\title{
Azimuthal dependence of the density distribution in outer galactic discs accreting intergalactic flows
}

\author{
M. López-Corredoira ${ }^{1}$ and J. Betancort-Rijo ${ }^{1,2}$ \\ ${ }^{1}$ Instituto de Astrofísica de Canarias, 38200 La Laguna, Tenerife, Spain \\ e-mail: martinlc@iac.es \\ 2 Departamento de Astrofísica, Universidad de La Laguna, Tenerife, Spain
}

Received 27 October 2008 / Accepted 20 November 2008

ABSTRACT

\begin{abstract}
Aims. The amplitude and scaleheight of the Galactic gas disc density are not axisymmetric against expectations in a self-gravity axisymmetric disc. However, this lopsidedness can be explained in terms of intergalactic accretion flows, which produce nonaxisymmetric pressure on the disc. This mechanism could be also responsible for the formation of a warp.

Methods. We analytically derive the relationship between the disc density and the self-gravity and external pressure.

Results. The same scenario of accretion as we proposed years ago to explain the formation of the warp explains the azimuthal dependence of the density and its scaleheight, with minimum/maximum in the positions of maximum amplitude of the warp $\left(\phi \approx 95^{\circ}\right.$ and $275^{\circ}$ ), as expected from its pressure distribution.
\end{abstract}

Key words. galaxies: kinematics and dynamics - galaxies: structure - Galaxy: structure

\section{Introduction}

There are several proofs that the scaleheight of the gas disc in our Galaxy depends both on the galactocentric distance (Narayan \& Jog 2002; Nakanishi \& Sofue 2003) and the azimuth (Voskes 1999; Levine et al. 2006; Kalberla et al. 2007). And there are explanations for the radial dependence of the scaleheight of galactic discs in spiral galaxies (flares) in terms of self-gravitating discs (e.g., Narayan \& Jog 2002) or magnetic fields (Battaner \& Florido 1995). However, it is not easy to understand the azimuthal dependence in the same terms, except for an exotic proposal of the existence of a ring of dark matter embedded in the galactic disc with a radius that depends on the azimuth (Kalberla et al. 2007). We think we have a better solution that is less exotic, less ad hoc, and that agrees with other observed galactic features, too.

In López-Corredoira et al. (2002, hereafter LBB), we proposed a mechanism to explain the formation of warps ( $\mathrm{S}$-shaped or U-shaped or a combination of both) in spiral galaxies: the accretion of the intergalactic medium (IGM) onto the disc (see LBB, Fig. 6). Indeed, up to now there have been no alternative explanations for the formation of U-warps. No massive halo is necessary, or high values of magnetic fields, or satellite companions are necessary, although the presence of these elements would not modify qualitatively the present conclusions. In a Milky-Way-like galaxy, the mean density of baryonic matter in the IGM needed to produce the observed warp is around $10^{-25} \mathrm{~kg} / \mathrm{m}^{3}$ when the infall velocity at a large distance is $\sim 100 \mathrm{~km} \mathrm{~s}^{-1}$ (LBB). These numbers were corroborated independently by Sánchez-Salcedo (2006). This hypothetical lowdensity net flow is a very reasonable physical assumption that would explain why most spiral galaxies are warped. There are also different types of observations successfully explained by the LBB hypothesis (López-Corredoira et al. 2008): 1) accretion of $\sim 1 M_{\odot} /$ yr of low metallicity gas onto the disc as expected from the chemical evolution of the Milky Way (see LBB, Sect. 3.1); 2) frequency of warps and its amplitude depending on environment; 3) lower frequency of U-warps over S-warps.

In this paper, we claim that the same mechanism proposed by LBB to explain the Galactic S-warp is able to explain another observational fact: the azimuthal dependence of the gas density and scaleheight in the outer disc.

\section{Density distribution in the vertical direction of a self-gravitating disc}

As has been known for a long time (Spitzer 1942; Narayan \& Jog 2002, and references therein), the distribution of matter in the vertical direction of the disc can be derived from applying the hydrostatic equilibrium,

$\frac{\left\langle v_{z}^{2}\right\rangle}{\rho} \frac{\partial \rho}{\partial z}=a_{z}$,

and the Poisson equation,

$\frac{\partial a_{z, \text { grav }}}{\partial z}=-4 \pi G \rho-\frac{1}{R} \frac{\partial\left(R a_{R, \text { grav }}\right)}{\partial R}-\frac{1}{R} \frac{\partial a_{\phi, \text { grav }}}{\partial \phi}$,

where $\rho$ is the mass density; $\left\langle v_{z}^{2}\right\rangle$ the variance of the vertical velocities along the vertical $z$ direction in an isothermal disc; $a_{R}$, $a_{\phi}$, and $a_{z}$ are the three components of the acceleration per unit mass in cylindrical coordinates $(R, \phi, z$; the vertical direction $z$ being defined as perpendicular to the ring); and $a_{x, \text { grav }}$ is the part of the $x$-component due to the self-gravity of the disc. On the right-hand side of Eq. (2), the third term is zero for an axisymmetric disc, and the second term is neglected for a thin-disc approximation, which are the normal assumptions in most analyses 
of the scaleheight. Here we do not neglect the second term since the scaleheight is not small. Instead, we adopt a monopolar approximation for the radial acceleration,

$a_{R, \mathrm{grav}} \approx \frac{-G M_{\mathrm{gal}}}{R^{2}}$,

where $M_{\text {gal }}$ is the mass of the Galaxy within radius $R$, which we consider constant for the outer disc. For the azimuthal acceleration $a_{\phi, \text { grav }}$, there is some contribution from the warp, but we did check that it is negligible. We integrated over all the rings of the warped galaxy with a height $z_{w}(R, \phi)$, which we take from Levine et al. (2006), and we found that the azimuthal accelerations are of the order of $10^{-13} \mathrm{~m} / \mathrm{s}^{2}$, and the third term of the right-hand side Eq. (2) is more than 50 times lower than the second term for all values of $R>15 \mathrm{kpc}$ and $\phi$. Therefore we can neglect this direct effect of the warp. The effect of the bar is also negligible: azimuthal accelerations lower than $5 \times 10^{-14} \mathrm{~m} / \mathrm{s}^{2}$ with the potential of the long bar of López-Corredoira (2007, Eq. (7)). Therefore, if one only considers the self-gravity acceleration, by doing the derivative with respect to $z$ of Eq. (1) and assuming that the gravity is the only acceleration dependent on $z$, with Eqs. (2) and (3), we get

$\rho^{\prime \prime}-\frac{\rho^{\prime 2}}{\rho}+K_{1} \rho^{2}+K_{2} \rho=0$,

where $K_{1}=\frac{4 \pi G}{\left\langle v_{z}^{2}\right\rangle}, K_{2}=\frac{G M_{\text {gal }}}{R^{3}\left\langle v_{z}^{2}\right\rangle}$. The prime (') stands for the derivative with respect to $z$.

\subsection{Solutions of the differential Eq. (4)}

The differential Eq. (4) may be expressed as an integral. If we set as a boundary condition that the maximum density is reached at $z=z_{0}\left(\rho\left(z_{0}\right)=A, \rho^{\prime}\left(z_{0}\right)=0\right)$, we obtain

$z=z_{0} \pm \int_{0}^{\frac{A-\rho(z)}{A}} \frac{\mathrm{d} x}{(1-x) \sqrt{2 K_{1} A x-2 K_{2} \ln (1-x)}}$

where $x=\frac{A-\rho}{A}$; which is a symmetrical distribution of $\rho(z)$ with respect to $z_{0}$. The half-width-half-maximum $(H W H M)$ is

$H W H M=\int_{0}^{1 / 2} \frac{\mathrm{d} x}{(1-x) \sqrt{2 K_{1} A x-2 K_{2} \ln (1-x)}}$,

and the surface density is

$\sigma=\int_{-\infty}^{\infty} \mathrm{d} z \rho(z)=2 A \int_{0}^{1} \frac{\mathrm{d} x}{\sqrt{2 K_{1} A x-2 K_{2} \ln (1-x)}}$.

For very thin discs ( $\rho$ very high), the second term on the righthand side of Eq. (2) is negligible $\left(K_{2} \approx 0\right)$. The solution to this differential equation with $K_{2}=0$ is the classical squared hyperbolic secant solution (Spitzer 1942): $\rho(z)=A \operatorname{sech}^{2}\left[a\left(z-z_{0}\right)\right]$, $a=\sqrt{A K_{1} / 2}=K_{1} \sigma / 4$.

Since $K_{1}$ and $K_{2}$ do not depend on $\phi$, it is clear that the width of the disc, $H W H M$, is independent of $\phi$, too, and only depends on the radius, so the self-gravity application does not explain the azimuthal dependence of the scaleheight.

\section{With external pressure by a continuous accretion of IGM}

We propose that the explanation for the variation in the gas disc thickness depending on $\phi$ is that the external pressure due to the accretion of IGM onto the disc depends on $\phi$, because the average flow falls down to the disc with an angle different to $\pi / 2$ in general with respect to the plane (LBB). The pressure exerted over the disc would be similar to a piston mechanism, only from one side of the disc (Sánchez-Salcedo 2006, Sect. 4.6). The action is perhaps ram-pressure, due to the friction of clouds against the interstellar medium (Sofue \& Wakamatsu 1993).

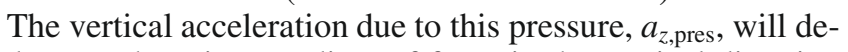
pend on $z$. There is a gradient of force in the vertical direction due the higher absorption of linear momentum by the first layers of the disc that collide with the accreted gas. For a very low dense, disc, as is the case of the very outer disc, the absorption of momentum is not total, because part of the gas can cross the disc completely and escape from it. We suppose that the external pressure is attenuated exponentially along the $z$-axis $P_{z, \text { ext }}(z)=P_{z \text {,ext }}( \pm \infty) \mathrm{e}^{ \pm C\left[\int_{ \pm \infty}^{z} \rho(z) \mathrm{d} z\right]}$, where $C$ is a cross-section per unit mass characteristic of the interaction between the accreted flow and the gas disc along the vertical axis. Here we do not take into account the effect of the galactic rotation and the variations in the attenuation with different incident angles. The sign + or depends on whether the flow comes from $z=\infty$ or $z=-\infty$. The force per unit volume is $-\nabla P_{z}(z)$. Hence,

$a_{z, \text { pres }}(R, \phi, z)=-( \pm) F_{z}(R, \phi) C \mathrm{e}^{ \pm C\left[\int_{ \pm \infty}^{z} \rho(z) \mathrm{d} z\right]}$,

where $F_{z} \equiv P_{z, \text { ext }}( \pm \infty)$ is the vertical component of the external force per unit surface $(\mathrm{d} S=R \mathrm{~d} R \mathrm{~d} \phi)$ due to accretion.

We do the derivative of Eq. (8), taking the exponential close to one in a low-density disc in the very outer disc $\left(\frac{1}{2} C \sigma\right.$ small compared to one; indeed, in Sect. 4 we will see that $\frac{1}{2} C \sigma<\sim$ $10^{-13}\left[\rho_{b}\left(\mathrm{~kg} / \mathrm{m}^{3}\right)\right]^{-1 / 2}$, which is smaller than one for the expected values of $\rho_{b}\left(\sim 10^{-25} \mathrm{~kg} / \mathrm{m}^{3}\right)$ although not much smaller; but we take this as a rough approximation):

$a_{z, \text { pres }}^{\prime} \approx-F_{z} C^{2} \rho$.

Given a continuous inflow of particles with density at infinite distance $\rho_{b}$, velocity $v_{0}$, and angle with respect to the plane $\theta_{0}$ with azimuth $\phi_{0}$ of the direction of the inflow (so the flow comes from $\left.-\theta_{0}, \phi_{0}+\pi\right)$, and Galactic mass within $R$ of $M_{\text {gal }}$, the following results hold (from LBB, Eq. (45); applying Eqs. (28), (33), (34), (38) of the same paper) ${ }^{1}$

$F_{z}(R, \phi) \mathrm{d} S=\frac{\rho_{b} v_{0}^{2} \mathrm{~d} S}{R^{2}\left|\sin \theta_{0}\right|} \frac{x_{1}}{\left(1+\sin ^{2}\left(\phi_{0}-\phi\right)\left(\frac{1}{\sin ^{2}\left(\theta_{0}\right)}-1\right)\right)}$

$\times\left[\frac{R x_{2}}{2}+\sqrt{\frac{R^{2} x_{2}^{2}}{4}+\frac{R G M_{\mathrm{gal}} x_{3}}{v_{0}^{2}}}\right]^{2} \times\left[\frac{x_{2}}{2}+\frac{\frac{R x_{2}^{2}}{4}+\frac{G M_{\mathrm{gal}} x_{3}}{2 v_{0}^{2}}}{\sqrt{\frac{R^{2} x_{2}^{2}}{4}+\frac{R G M_{\mathrm{gal}} x_{3}}{v_{0}^{2}}}}\right]$,

$x_{1}(\phi)=\sqrt{1-\cos ^{2} \theta_{0} \sin ^{2}\left(\phi_{0}-\phi\right)}$

$x_{2}(\phi)=\sqrt{1-\cos ^{2} \theta_{0} \cos ^{2}\left(\phi_{0}-\phi\right)}$

$x_{3}(\phi)=1+\cos \theta_{0} \cos \left(\phi_{0}-\phi\right)$.

Figure 1 plots $F_{z}^{-1}$ with the parameters used by LBB: $M_{\text {gal }}=$ $2 \times 10^{11} M_{\odot}, v_{0}=100 \mathrm{~km} \mathrm{~s}^{-1}$, and $\theta_{0}$ is a free parameter. $F_{z}$

1 We have found an erratum in LBB: Eq. (28) should have opposite sign, i.e. $e_{0 Q}=-\cos \left(\boldsymbol{v}_{\mathbf{0}}, \boldsymbol{r}_{Q}\right)=-\cos \left(\theta_{0}\right) \cos \left(\phi_{0}-\phi\right)$. However, numbers and orientation of the warp are correct as stated in LBB. 


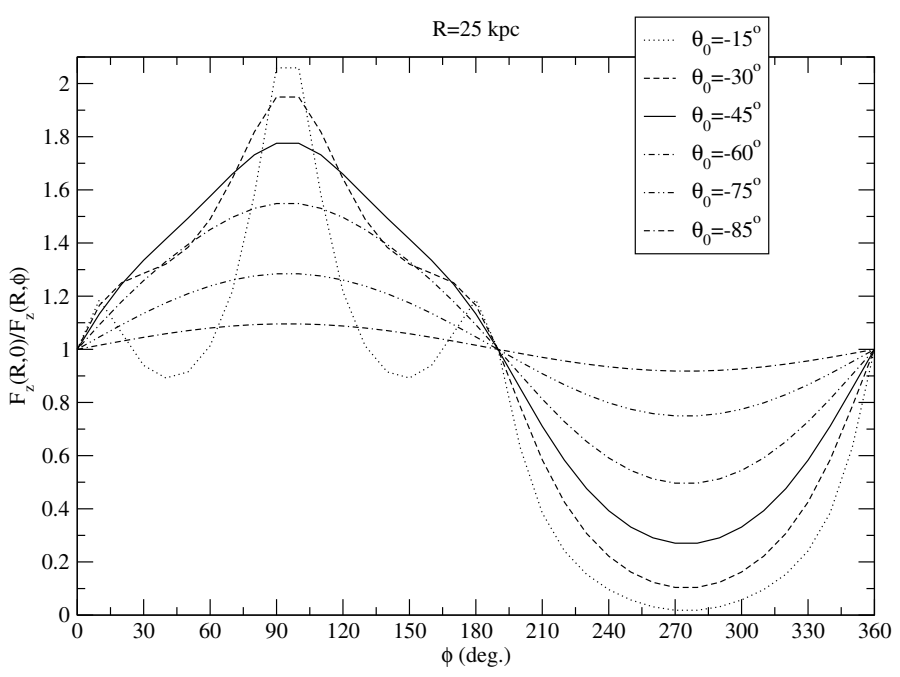

Fig. 1. $F_{z}^{-1}$ according to Eq. (10) with the parameters given by LBB and $\phi_{0}=275^{\circ}$.

is $\sim 10^{-14} \mathrm{~kg} / \mathrm{m} / \mathrm{s}^{2}$. We take $\phi_{0}=275^{\circ}, \theta_{0}<0$, the corresponding direction of the accreted flow, in order to produce the Galactic S-warp with U-warp southwards (LBB) and maximum of the S-warp at $\phi=95^{\circ}, 275^{\circ}$, as observed (Voskes 1999). The dependence is plotted for $R=25 \mathrm{kpc}$ and variable $\phi$, while other values of $R$ give different amplitudes in the variation, but similar azimuthal dependence. The amplitude of the variation with the azimuth is strongly dependent on $\theta_{0}$. Clearly, for any value of $\theta_{0}$, the minimum pressure is for $\phi=\phi_{0}+\pi=95^{\circ}$, and the maximum is for $\phi=\phi_{0}=275^{\circ}$. Nonetheless, if we took into account the rotation of the galaxy and an external pressure attenuation dependent on this and the incident angle, or the degree of clumpiness of IGM and disc, the difference in the position of the maximum and minimum would not be strictly $\pi$, and the shape of Fig. 1 would vary.

\subsection{Differential equation}

If we now consider that the total acceleration is $a_{z}=a_{z \text {,grav }}+$ $a_{z \text {,pres }}$ in Eq. (1), assuming an immediate response of the disc to the pressure exerted by the accreted material (López-Corredoira et al. 2008), together with Eqs. (2) and (9), we again get the same differential Eq. (4) but with constants $K_{1}=\frac{4 \pi G+F_{z}(R, \phi) C^{2}}{\left\langle v_{z}^{2}\right\rangle(R, \phi)}$, $K_{2}=\frac{G M_{\mathrm{gal}}}{R^{3}\left\langle v_{z}^{2}\right\rangle(R, \phi)}$. The total acceleration is null at $z_{0}$, where the pressure acceleration is compensated by other gravitational forces to keep the warp static. With $C \sim 10^{2}-10^{3} \mathrm{~m}^{2} / \mathrm{kg}$, we get values of $F_{z}(R, \phi) C^{2}$ of the order of $4 \pi G$, that is, an external pressure as significant as the self-gravity. And, most important, $H W H M$ will depend on $\phi$ because $F_{z}$ depends on $\phi$ and also on the maximum amplitude of the density, $A$, and the dispersion of velocities depends on $\phi$ through its dependence on the pressure at $z=z_{0} P(\phi)$ :

$A(\phi)=A\left(\phi_{0}\right)\left(\frac{P(\phi)}{P\left(\phi_{0}\right)}\right)^{\frac{1}{\gamma}}$,

$\left\langle v_{z}^{2}\right\rangle(\phi)=\left\langle v_{z}^{2}\right\rangle\left(\phi_{0}\right)\left(\frac{P(\phi)}{P\left(\phi_{0}\right)}\right)^{\frac{\gamma-1}{\gamma}}$

$P(\phi) \propto\left(1+\frac{F_{z}(\phi) C^{2}}{4 \pi G}\right)$.

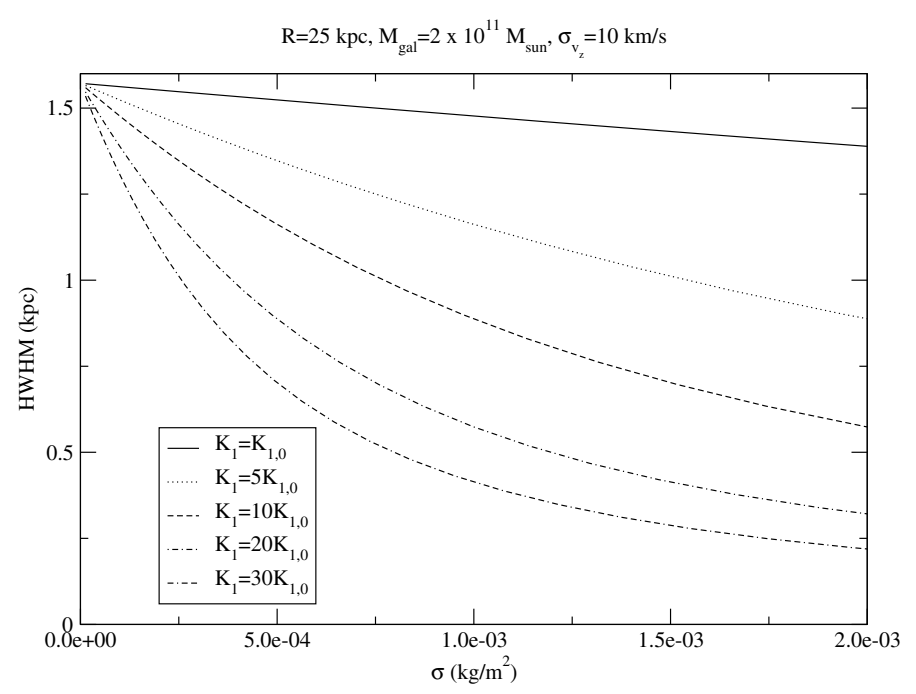

Fig. 2. Dependence of $H W H M$ on $\sigma$, from Eqs. (6) and (7), for different values of $K_{1}$ with the minimum value $K_{1,0}=\frac{4 \pi G}{\left\langle v_{2}^{2}\right\rangle}$ given when only gravity is present and other values when an external pressure is added, and fixed $K_{2}$ and $\left\langle v_{z}^{2}\right\rangle$.

This last proportionality of the pressure stems from the external pressure acting like an extra self-gravity, and the pressure is proportional to the total acceleration. For a monoatomic gas, $\gamma=5 / 3$ for adiabatic compression, and $\gamma=1$ for isothermal one. This applies in the distribution of pressure as a function of $\phi$; for the vertical dependence, we have already assumed that it is isothermal.

In Fig. 2, we see how the $H W H M$ is reduced for a given $\sigma$ when $K_{1}$ is increased, i.e. when $F_{z}$ is increased. The parameters are $R=25 \mathrm{kpc}, M_{\mathrm{gal}}=2 \times 10^{11} M_{\odot}$, and $\sigma_{v_{z}}=10 \mathrm{~km} \mathrm{~s}^{-1}$.

\section{Comparison with the observations}

Voskes (1999, Fig. 15) and Levine et al. (2006, Fig. 5) have shown that the scaleheight of the outer disc $(R>20 \mathrm{kpc})$ is $2-3$ times higher on average for $0<\phi<180^{\circ}$ than for $180^{\circ}<\phi<360^{\circ}$. A more accurate estimation of the maximum of the scaleheight is derived by Kalberla et al. (2007, Figs. 18, 19), who place it for $90^{\circ}<\phi<110^{\circ}$, while the minimum for $250^{\circ}<\phi<270^{\circ}$. Within the LBB scenario, two possible directions are possible to produce the observed S-warp, and this is one of them: the wind coming from the direction of the northern warp, although this solution could not explain the asymmetry of the southern/northern warp as a sum of S+U warp. A higher pressure is expected for the region around the southern warp and consequently a lower thickness therein. Not only is the pressure lower at $\phi \approx 90^{\circ}$, but the surface density $\sigma$ is also lower than for the average value of $\sigma(R)$ (Voskes 1999, Fig. 13; Levine et al. 2006, Fig. 1; Kalberla \& Dedes 2008, Fig. 9) and the amplitude of the density $A$ (Kalberla \& Dedes 2008, Fig. 8). This is another fact explained by our model by means of Eqs. (7) and (11): the lower the pressure the lower the density.

If we take the values observed by Kalberla et al. $(2007,2008)$ at $R=25 \mathrm{kpc}$, excluding the bin of $90^{\circ}<\phi<110^{\circ}$, which is possibly an outlier in a special region: $A\left(\phi_{0}\right)=1.5 \times 10^{-24} \mathrm{~kg} / \mathrm{m}^{3}$; $\frac{A\left(\phi_{0}+\pi\right)}{A\left(\phi_{0}\right)} \approx 7, \frac{\sigma\left(\phi_{0}+\pi\right)}{\sigma\left(\phi_{0}\right)} \approx 4, \frac{H W H M\left(\phi_{0}\right)}{H W H M\left(\phi_{0}+\pi\right)}=2.5$. This last number is not independent of the other three. The parameters that better fit these numbers are: $\gamma=1.22, \theta_{0}=-31^{\circ}, \rho_{b} C^{2}=$ $1.8 \times 10^{-19} \mathrm{~m} / \mathrm{kg}$, giving an azimuthal dependence of $H W H M, A$ and $\sigma$ as plotted in Fig. 3. The average accretion at $R=25 \mathrm{kpc}$ 


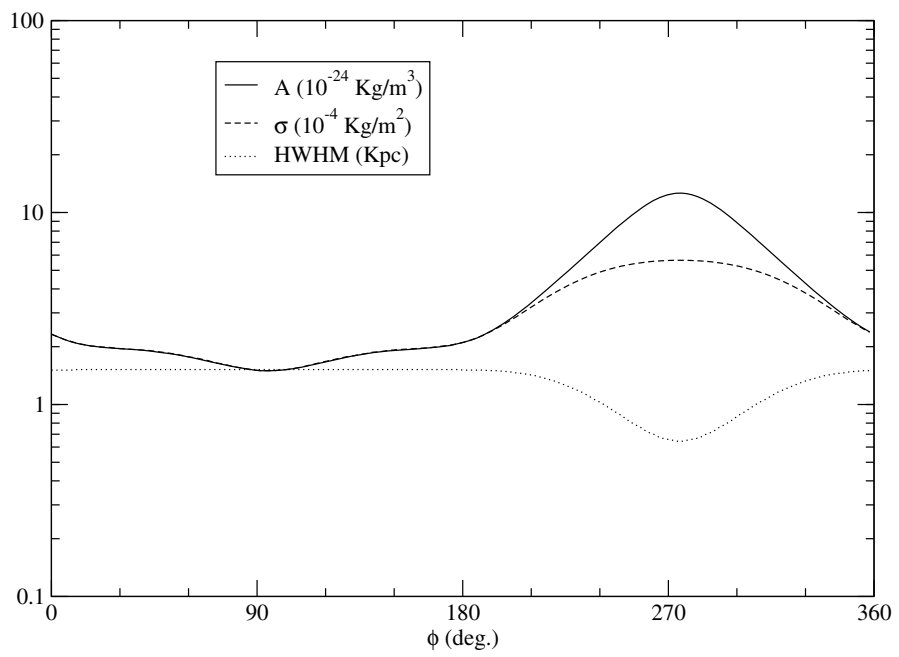

Fig. 3. Azimuthal dependence of the variables $H W H M, A$ and $\sigma$ for $R=$ $25 \mathrm{kpc}$. Parameters: $v_{0}=100 \mathrm{~km} \mathrm{~s}^{-1}, M_{\text {gal }}=2 \times 10^{11} M_{\odot}, \sigma_{v_{z}}\left(\phi_{0}\right)=$ $10 \mathrm{~km} \mathrm{~s}^{-1}, A\left(\phi_{0}\right)=1.5 \times 10^{-24} \mathrm{~kg} / \mathrm{m}^{3}, \phi_{0}=275^{\circ} ; \gamma=1.22, \theta_{0}=-31^{\circ}$, $\rho_{b} C^{2}=1.8 \times 10^{-19} \mathrm{~m} / \mathrm{kg}$.

is $5 \times 10^{-4}\left(\frac{\rho_{b}}{10^{-25} \mathrm{~kg} / \mathrm{m}^{3}}\right) \mathrm{kg} / \mathrm{m}^{2} / \mathrm{Gyr}$, which is around 2 times the average surface density per Gyr; note, however, that only a small ratio of its linear momentum $(\sim C \sigma)$ is transmitted to the Galactic disc.

For the shape of the scaleheight as a function of $\phi$ for $R=25 \mathrm{kpc}$ (or any other value of the galactocentric distance $20 \leq R \leq 40 \mathrm{kpc}$ ), we observe in Fig. 19 of Kalberla et al. (2007) a strongest dependence of the scaleheight with $\phi$ near the maximum; however, the variation in amplitude is very slight around the minimum of flaring. This is not observed in the shape of Fig. 3.

Therefore, at present with our simple model, we cannot explain the exact azimuthal dependence. Possibly, our rough assumptions in the present and LBB calculations need to be improved to get a more accurate result, assuming that Kalberla et al. $(2007,2008)$ are also correct with their assumptions on the kinematics of the Galaxy. Indeed, the Kalberla et al. (2007, 2008) density distributions are derived by assuming a nonaxisymmetric disc using epicyclic corrections in the rotation curve, consistent with their hypothesis to explain the asymmetries in the outer disc. Since we can explain the asymmetries of the outer disc with the accretion of IGM flows, it is possible that those epicyclic corrections are not necessary, although they are not necessarily inconsistent with our scenario. In any case, our model describes the asymmetries roughly. The value of the parameters for the infall material also more or less agree the values necessary for the warp production in LBB. If Kalberla et al. assumed axisymmetric rotation, they would get a gas distribution that is several $\mathrm{kpc}$ more extended to the south $\left(180^{\circ}<l<360^{\circ}\right)$ than to the north (Levine et al. 2006, Fig. A18). This would also agree with our scenario because we predict a higher density in the south in general.

\section{Conclusions}

The same LBB scenario that explains the formation of warps is able to directly explain the azimuthal dependence of the HI density distribution in the Milky Way, with maximum and minimum in the positions expected, without introducing ad hoc elements. Sánchez-Salcedo (2006) raises the criticism that LBB mechanism is not plausible because it would produce a dependence on the scaleheight of the disc with the Galactocentric azimuth, $\phi$, in the outer disc. Rather than being an objection, it is another argument in favour of our model because this dependence is actually observed in our Galaxy.

Acknowledgements. Thanks are given to the anonymous referee for helpful comments, and to Joly Adams (language editor of A\&A) for proof-reading this paper. M.L.C. was supported by the Ramón y Cajal Programme and the grant AYA2007-67625-CO2-01 of the Spanish Science Ministery.

\section{References}

Battaner, E., \& Florido, E. 1995, MNRAS, 277, 1129

Kalberla, P. M. W., \& Dedes, L. 2008, A\&A, 487, 951

Kalberla, P. M. W., Dedes, L., Kerp, J., \& Haud, U. 2007, A\&A, 469, 511

Levine, E. S., Blitz, L., \& Heiles, C. 2006, ApJ, 643, 881

López-Corredoira, M. 2007, A\&A, 469, 471

López-Corredoira, M., Betancort-Rijo, J., \& Beckman, J. E. 2002, A\&A, 386, 169 [LBB]

López-Corredoira, M., Betancort-Rijo, J., \& Beckman, J. E. 2008, in Pathways Through an Eclectic Universe, ed. J. H. Knapen, T. J. Mahoney, \& A. Vazdekis, (S. Francisco: ASP), ASP Conf. Ser., 390, 359

Nakanishi, H., \& Sofue, Y. 2003, PASJ, 55, 191

Narayan, C. A., \& Jog, C. J. 2002, A\&A, 394, 89

Sánchez-Salcedo, F. J. 2006, MNRAS, 365, 555

Spitzer, L. 1942, ApJ, 95, 329

Sofue, Y, \& Wakamatsu, K. 1993, A\&A, 273, 79

Voskes, T. 1999, M.Sc. thesis, Univ. Leiden [arXiv: astro-ph/0601653] 